\title{
Problems in event based engine control
}

Hendricks, Elbert; Jensen, Michael; Chevalier, Alain Marie Roger; Vesterholm, Thomas

\section{Published in:}

Proceedings of the American Control Conference

Link to article, DOI:

10.1109/ACC. 1994.752337

Publication date:

1994

Document Version

Publisher's PDF, also known as Version of record

Link back to DTU Orbit

Citation (APA):

Hendricks, E., Jensen, M., Chevalier, A. M. R., \& Vesterholm, T. (1994). Problems in event based engine control. In Proceedings of the American Control Conference (Vol. Volume 2, pp. 1585-1587). IEEE. https://doi.org/10.1109/ACC.1994.752337

\section{General rights}

Copyright and moral rights for the publications made accessible in the public portal are retained by the authors and/or other copyright owners and it is a condition of accessing publications that users recognise and abide by the legal requirements associated with these rights.

- Users may download and print one copy of any publication from the public portal for the purpose of private study or research.

- You may not further distribute the material or use it for any profit-making activity or commercial gain

- You may freely distribute the URL identifying the publication in the public portal

If you believe that this document breaches copyright please contact us providing details, and we will remove access to the work immediately and investigate your claim. 


\title{
Problems in Event Based Engine Control
}

\author{
Elbert Hendricks", Michael Jensen ${ }^{* *}$, Alain Chevalier" and Thomas Vesterholm* \\ "Institute of Automatic Control Systems \\ *"Laboratory for Energetics \\ The Technical University of Denmark \\ DK-2800 Lyngby, Denmark \\ e-mail eh@sl.dth.dk
}

\begin{abstract}
Physically a four cycle spark ignition engine operates on the basis of four engine processes or events: intake, compression, ignition (or expansion) and exhaust. These events each occupy approximately $180^{\circ}$ of crank angle and in conventional engine controllers, it is an accepted practice to sample the engine variables synchronously with these events (or submultiples of them). Such engine controllers are often called event based systems.
\end{abstract}

Unfortunately the main system noise (or disturbance) is also synchronous with the engine events: the engine pumping fluctuations. Since many electronic engine controllers are event sampled systems, the sampling frequency is thus also frequency locked to the main system disturbances. This creates a signal analysis problem which has, to the authors' knowledge, not been treated in detail in the literature. This paper concerns itself with the impact of this and related problems on accurate air/fuel ratio control of a spark ignition (SI) engine.

\section{Introduction}

Microprocessor controllers for spark ignition (SI) as they currently exist are a natural consequence of the physical understanding of engine behavior which existed when they were first designed, i. e., in about 1980. This has had a great effect on their configuration.

For example, the first generation engine controllers currently in production often sample the data from their sensors on an event (or constant crank angle increment basis) because the basic reciprocating engine process works in this way [1]. For this reason many controller operating systems are event interrupt driven. This has appeared to be a reasonable design approach but interestingly a reasonably logical and complete analysis of such a system has, to the authors' knowledge, never appeared in the literature.

The purpose of this paper is to address the overall engine sensor/measurement problem in an attempt to define what the characteristics and limitations of event based control are. No general attack on event based sampling is intended, only an analysis of systems already in use. The main systems of interest here will be those which have a mechanical connection between the accelerator pedal and throttle plate. Results to be presented will however also impact more advanced (f. ex., observer based) control systems as well as those which use electronic throttle control.

\section{System Input and State Variables}

In order to focus attention on the overall measurement problem, figure 1 is presented. This figure shows schematically the basic elements of an engine control system. The engine sensors (including the throttle position potentiometer) feed signals to the control microprocessor via antialiasing filters, sample and hold networks and A/D convertor(s). These signals are processed in the computer and signals output to the system actuators (the spark drives and fuel injector(s)). The engine sensors then feed information about the engine state back into the control microprocessor. The sensor

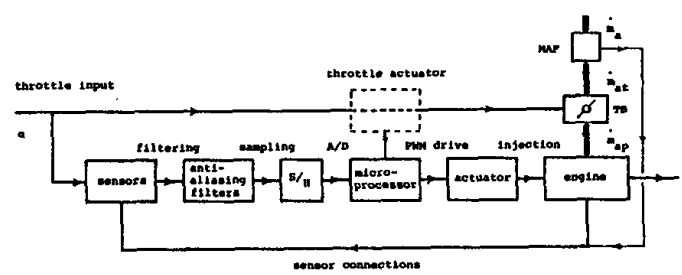

Figure 1. Schematic block diagram of an engine control system showing its main measurement components. 
measurement problem is then to feed the operator's commands to the throttle plate (and the resulting senor signals) through the measurement input system to the engine control microprocessor.

\section{System Analysis in the Frequency Domain}

A simple, approximate model for the operator's commands is a low pass filtered white noise signal with a bandwidth equal to the reciprocal of the operator's minimum throttle movement time (about $50 \mathrm{msec}$ ). This frequency domain picture gives a natural way of treating the sampling and aliasing problems in the measurement chain.

From the above, it is reasonable to approximate the operator input as low pass filtered white noise which has a cut off frequency of about $20 \mathrm{~Hz}$. From the analysis of a Mean Value Engine Model (MVEM) developed earlier for an SI engine [2], it is known that the air mass flow related variables which are usually measured in an engine controller (such as the manifold pressure or the air mass flow) will change on the same time scale as the throttle input [3]. A representative spectrum for the throttle angle or for an air mass flow related sensor signal is shown on figure $2 a$, where $\alpha$ is the throttle angle, $\mathrm{p}_{\mathrm{m}}$ is the manifold pressure and $\mathrm{m}_{\mathrm{a}}$ is the air mass flow measured in front of the throttle plate. For proper operation of an engine control system, this input/sensor spectrum must be faithfully passed into the engine control microprocessor. Note that linear amplitude and frequency axes have been used on the plot and all variables have been (and will be) normalized in such a way that they are suitably scaled and are dimensionless for convenience of presentation.

In a real engine control system, the air mass flow related signals will have a strong noise component due to engine pumping fluctuations. This noise will be concentrated at the engine event frequency. For the sake of illustration a four cylinder, four cycle engine will be considered in what follows. Such an engine, running at between $n=600$ and $6000 \mathrm{rpm}$ will have an event frequency between 20 and 200 Hz. This will give rise to a deterministic noise signal at the event frequency on the air mass flow related sensor signals as suggested on figure $\mathbf{2 a}$ (only the first harmonic of the event disturbance is considered).

The spectrum in figure $2 a$ is that which the engine sensors must reproduce. For comparison with this spectrum, typical engine sensor response characteristics are reproduced in figure $2 \mathrm{~b}$. A typical MAP (Manifold Absolute Pressure) sensor has a first order low pass response with a cut-off frequency of
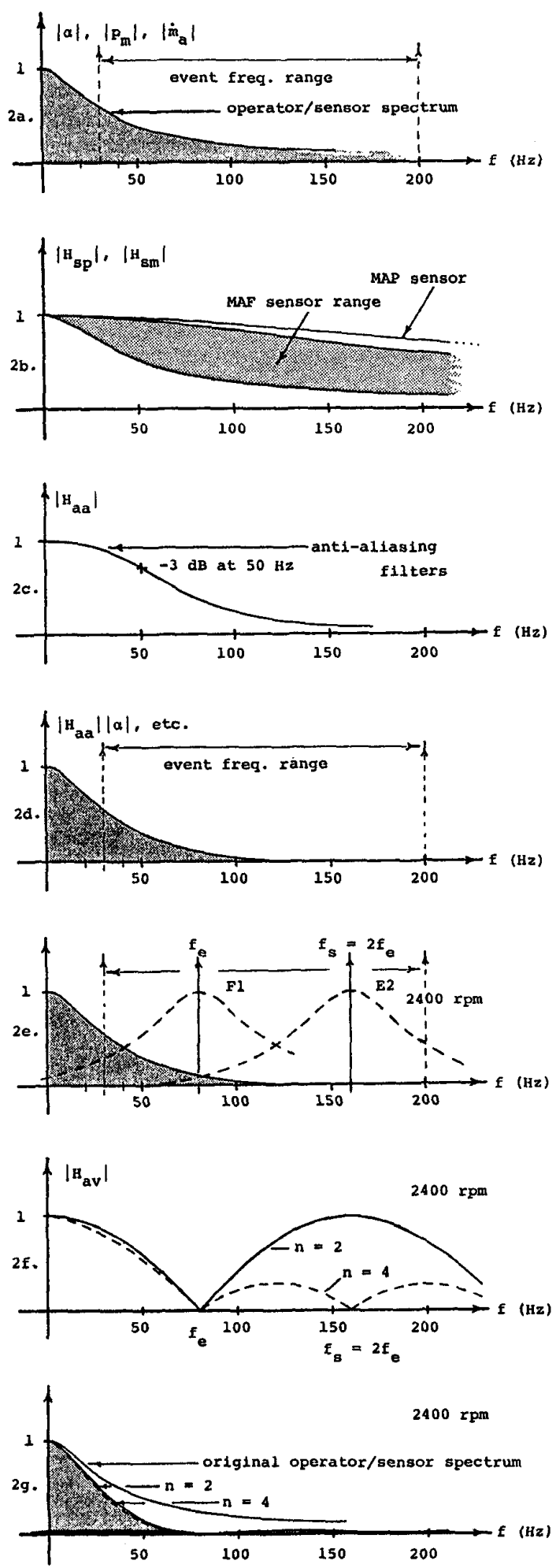

Figure 2. A system analysis of an engine control system in the frequency domain. The different parts of the figure show how the operator's input and resulting signal spectra are propagated through the measurement chain. 
about $200 \mathrm{~Hz}$. A typical bobbin hot wire MAF (Mass Air Flow) sensor has a variable small signal cut-off frequency of between 40 and $150 \mathrm{~Hz}$, depending on the operating point. From figures $2 \mathrm{a}$ and $2 b$ it is clear that only the MAP sensor is fast enough to pass the actual operator input spectrum into the engine control microprocessor. The MAF sensor is only marginally fast enough. From this point the response time of the air mass flow related sensors will not be considered further in this paper.

\subsection{Overall System Response}

In order to reduce high frequency noise it is common to introduce an anti-aliasing filter into the measurement chain before the sensor signal is sampled. Such a filter (second order with a cut-off frequency of $50 \mathrm{~Hz}$ ) is shown on figure $2 \mathrm{c}$ and its effect on the operator input or sensor(s) spectrum is shown on figure $2 d$.

After the signals have been low pass filtered, they can be sampled in the sample and hold networks and/or A/D convertors. In general this is done in a very short time interval (relative to an event period) at one or more times per engine event in an event based system. If the operator input spectrum is sampled once (F1) or twice per engine event (E2) the resulting spectrum is as shown in figure $2 \mathrm{e}$ at $\mathbf{n}$ $=2400 \mathrm{rpm}$. Here it is apparent that even at the relatively high crank shaft speed of $2400 \mathrm{rpm}$ there is a significant overlap between the original and sample spectra and in other words there is a strong possibility of aliasing. At lower crank shaft speeds and especially close to idle, the aliasing problem will be even worse.

All conventional engine controllers are based on finding the mean air mass flow to an engine (or equivalently, the mean air charge per stroke). This means that the it is necessary to suppress engine pumping fluctuations in the measurements. This can be done either by attempting to sample at exactly the correct phase in an engine revolution or by averaging several measurements during an event. The first sampling method might be called a Fixed phase Event Based Sampling (FEBSn or Fn algorithm, where $\mathrm{n}$ is the number of samples per event) while the second could be called an Event Averaged Based Sampling (EABSn or En algorithm, where $n$ is again the number of samples per event).

From figure $2 \mathrm{e}$ it is clear that the $\mathrm{Fl}$ algorithm can display serious aliasing even up to very high crank shaft speeds. Such an algorithm also is sensitive to phase changes due to resonances and acoustical effects as well as the signal waveform [3].
Averaging filters (En algorithms) have a different method of suppressing engine pumping fluctuations. Such filters corresponds to using a digital integrator on the sampled signal (with $n$ samples averaged per event). Figure $2 \mathrm{f}$ shows the frequency response of the averaging process for two (E2) or four (E4) samples per event. Clearly both filters can suppress disturbances at the event frequency. The higher the order of the averaging, the more harmonics of the event disturbance will be suppressed. Figure $2 \mathrm{e}$ shows however that there will be aliasing below about $2400 \mathrm{rpm}$ with the E2 algorithm. There are also problems with complex signal waveforms and with the inherent time delay in the averaging process [3].

The overall response of the system to the operator input spectrum is shown on figure $2 \mathrm{~g}$ where it is compared to original spectrum for the E2 and E4 algorithms. Clearly the bandwidth of the measurement system is less than it should be merely because of the sampling process itself. It is also true that the aliasing is already present in the microprocessor input signal at the moment of sampling (figure 2e) and is thus not suppressed by an En algorithm.

\section{Conclusions}

In conventional engine control systems, event based sampling in engine controllers can lead to serious aliasing problems, especially at low to medium engine speeds. This aliasing is especially significant at low to medium engine speeds. Event based sampling plus averaging decreases the effect of engine pumping disturbances but gives a measurement bandwidth reduction. Both of these effects can lead to significant $A / F$ ratio control problems. The results presented suggest that engine sensors should be sampled with different methods, depending on their function in the engine controller.

\section{References}

[1] E. Hendricks, P. Kaidantzis, P. Rasmussen, M. Jensen and T. Vesterholm, "Transient A/F Ratio Errors in Conven-tional SI Engine Controllers", SAE Technical Paper No. 930856, 1993.

[2] E. Hendricks and T. Vesterholm, "The Analysis of Mean Value SI Engine Models", SAE Technical Paper No. 920682, 1992.

[3] E. Hendricks, M. Jensen, A. Chevalier and T. Vesterholm, "Conventional Event Based Engine Control", SAE Technical Paper No. 940378, in Sp$1029,1994$. 